\title{
Interculturalidad e inclusión del alumnado marroquí en educación primaria de Andalucía
}

\author{
Teresa Terrón-Caro' \\ Verónica Cobano-Delgado Palma"
}

\section{Resumen}

En este trabajo se muestran los resultados de una investigación desarrollada en una de las zonas prioritarias para el asentamiento de población extranjera en España, concretamente la Comunidad Autónoma de Andalucía. En el curso 2011-2012, había en la región de estudio un total de 32.308 alumnos extranjeros matriculados en régimen general en Educación Primaria. En este sentido, se plantea el siguiente problema de investigación: ¿en qué medida se siente integrado el alumnado de origen marroquí de educación obligatoria en Andalucía? y ¿cuáles podrían ser las líneas de actuación para favorecer la convivencia escolar? Con el propósito de dar respuesta al problema planteado, el objetivo general de esta investigación es analizar aquellos factores más relevantes que favorecen la inclusión del alumnado de origen marroquí matriculado en centros de educación primaria públicos de Andalucía. La metodología utilizada es múltiple y diversa, combinando el enfoque cualitativo y cuantitativo. Se han empleado varias técnicas de recogida de información: el análisis documental, el cuestionario y la observación participante. Entre los principales resultados hallados, cabe destacar que el mayor obstáculo que el alumnado ha encontrado al llegar a España en su proceso de integración es la lengua. No obstante, la mayor parte del alumnado estudiado afırma tener una identidad múltiple, definiéndose a sí mismo tanto marroquí como español. Esta realidad demanda por parte de los educadores intervenciones pedagógicas planificadas para seguir favoreciendo y madurando las identidades múltiples, así como para mejorar la adquisición de la lengua de la escuela, instrumento clave para favorecer la inclusión.

\section{Palabras clave}

Inclusión - Interculturalidad - Educación Primaria -

Contacto: mttercar@upo.es

II- Universidad de Sevilla,

Sevilla, España.

Contacto: cobano@us.es 


\title{
Interculturalism and inclusion of Moroccan students in primary education in Andalusia
}

Teresa Terrón-Caro'

Verónica Cobano-Delgado Palmal

\begin{abstract}
This work brings the results of a study carried out in one of the priority areas for the settlement of foreign population in Spain, namely Comunidad Autónoma de Andalucía. In the 2011-2012 academic year, there was a total of 32,308 foreign students enrolled in the general system of primary education in the region of study. In this regard, the following research question arises: to what extent do students of Moroccan origin in compulsory education in Andalusia feel integrated? And what might the lines of action to favor school coexistence be? In order to respond to the problem, the general objective of this research is to analyze the most relevant factors which promote the inclusion of students of Moroccan origin enrolled in public primary schools in Andalusia. The methodology used is multiple and diverse, combining quantitative and qualitative approaches. Several techniques have been used to gather information: documentary analysis, a questionnaire and participant observation. One of our main findings is that, when students arrive in Spain, the biggest obstacle to their integration process is language. However, most of the students claim to have a multiple identity, defining themselves both as Moroccan and Spanish. This reality demands from educators pedagogical interventions planned to further support and mature multiple identities, as well as to enhance the acquisition of the school language, which is a key tool to foster inclusion.
\end{abstract}

\section{Keywords}

Inclusion - Interculturalism - Primary education Immigration - Spain. 


\section{Introducción}

En este trabajo presentamos los resultados de una investigación ${ }^{1}$ desarrollada en la Comunidad Autónoma de Andalucía (España), con la finalidad de conocer la percepción que tiene el alumnado inmigrante de nacionalidad marroquí que cursa educación primaria ${ }^{2}$ acerca de su inclusión socioeducativa. En España, al igual que ocurre en otros países, existen algunas regiones que, por sus condiciones socioeconómicas, se han convertido en zonas prioritarias para el asentamiento de población extranjera, entre las que se encuentra Andalucía.

Es precisamente en estas regiones que cuentan con una densidad de población inmigrante relevante, donde las distancias culturales entre los colectivos de inmigrantes de determinadas nacionalidades y los grupos sociales autóctonos pueden conducir, si no existe comunicación entre ambos, a que se den actitudes cargadas de estereotipos, xenofobia. Esta falta de integración entre los distintos grupos culturales puede propiciar el fenómeno cada vez más habitual de los guetos culturales.

Debido a la amplitud y globalidad que caracteriza el fenómeno migratorio, puede ser analizado desde múltiples y diversas perspectivas. $\mathrm{Al}$ estudiarlo desde un prisma socioeducativo, la escuela se entorna protagonista, a la vez que se conforma como promotora de una sociedad verdaderamente intercultural.

Según los datos ofrecidos por el Observatorio Permanente Andalúz de las Migraciones (OPAM), para el curso 2011-2012 en Andalucía había un total de 32.308 alumnos extranjeros matriculados en régimen general en educación primaria, representando el 36,57\% del alumnado extranjero matriculado en las

1- El presente artículo es fruto del proyecto de investigación Integración y desarrollo colectivo inmigrante marroquí en Andalucía. Resolución de 12/11/2007, Consejería de Innovación, Ciencia y Empresa de la Junta de Andalucía. Desarrollado durante los años 2008, 2009 y 2010.

2- En España esta etapa educativa comprende tres ciclos de dos años cada uno y que se seguirán ordinariamente desde los seis hasta los doce años. Está organizada en áreas con carácter global e integrador. Tiene carácter obligatorio y gratuito. enseñanzas no universitarias. Si atendemos a las nacionalidades de procedencia, comprobamos que la marroquí es la principal sobre el total de alumnado extranjero matriculado en enseñanzas no universitarias $(23,5 \%)$, a la que le sigue Rumanía (13,8\%) y Reino Unido (7,8\%), entre otras. Por este motivo y por la gran distancia cultural existente entre la cultura española y la marroquí, consideramos importante conocer la opinión del alumnado originario de Marruecos respecto a la atención a la diversidad en las aulas de Andalucía.

No obstante, y antes de adentrarnos en la parte metodológica, así como en el análisis y discusión de los resultados obtenidos en nuestro estudio, consideramos importante partir de determinadas concepciones teóricas que ayuden a entender el trasfondo de las reflexiones aquí vertidas.

En primer lugar, debemos poner de manifiesto que entendemos la educación intercultural desde un enfoque holístico, presente en todo momento en el proceso de enseñanza-aprendizaje y dirigida a toda la comunidad educativa. Desde esta perspectiva, la educación intercultural es una oportunidad para la transformación de la institución educativa hacia una escuela inclusiva. Raquel Borrero nos dice que sólo desde un modelo educativo inclusivo se comprende la diversidad como una oportunidad para "el aprendizaje, el desarrollo, el crecimiento y la mejora. Y sólo desde este modelo educativo pueden formarse docentes preparados para gestionar la diversidad, sentirla como un valor positivo y entenderla como un bien común" (2012, p. 345). Ciertamente, este planteamiento exige nuevos retos trascendentales para el Estado, las administraciones con competencia en materia educativa, la institución educativa y la comunidad. Pues debemos pasar de la premisa de que todos somos iguales, en la que se pretende la normalización, al supuesto de que todos somos diferentes otorgándole a la diferencia un gran valor y una posibilidad de enriquecimiento. La escuela inclusiva debe 
enseñar "el igual valor de todas las diferencias personales, que hacen de cada persona un individuo diferente a todos/as los/las demás y a cada individuo una persona igual a las/los otras/os, con capacidad de enriquecerse de la heterogeneidad de los/as demás" (INADI, 2012, p. 9-10). Desde este paradigma, es fundamental comprender el concepto de identidad de una forma dinámica, siempre en continua construcción, donde las experiencias de vida de cada persona y las relaciones establecidas con otras culturas y grupos son elementales (ESCARBAJAL, 2011).

En segundo lugar, es preciso detenernos en analizar los términos que integran el concepto de diversidad cultural tan presente en nuestra sociedad y, particularmente, en el ámbito educativo. Por un lado, debemos ser conscientes de que: "La diversidad es una característica inherente a la naturaleza humana y una posibilidad para la mejora y el enriquecimiento de las condiciones y relaciones sociales y culturales entre las personas y entre los grupos sociales" (JIMÉNEZ; VILÁ, 1999). La diversidad es una realidad, un hecho concreto que atiende a la naturaleza misma. Es identificar a las personas tal y como son y, no como deberían ser. Sin embargo, nosotros tendemos a categorizar, nombrar y valorar esa diversidad biológica, lo que implica una apreciación subjetiva. Es en este momento cuando pasa a entenderse como diferencia, designando así una realidad de pensamiento, una construcción cognitiva acerca de la diversidad (PULIDO, 1997).

Por otro lado, y con la intención de compendiar otro de los términos básicos para abordar el tema que nos ocupa, nos adherimos a la definición de cultura propuesta por la UNESCO, en su Declaración Universal a favor de la diversidad cultural. Pues en ella se expresa que:

La cultura debe ser considerada como el conjunto de los rasgos distintivos espirituales y materiales, intelectuales y afectivos que caracterizan a una sociedad o a un grupo social y que abarca, además de las artes y las letras, los modos de vida, las maneras de vivir juntos, los sistemas de valores, las tradiciones y las creencias. (UNESCO $\left.{ }^{3}, 2001\right)$.

Por ello, la diversidad cultural, tan presente en nuestras sociedades, debe ser entendida como un aspecto enriquecedor para todos, tanto para nuestras generaciones como para las futuras. Debe ser reconocida y valorada como patrimonio de la humanidad. Y, al mismo tiempo, debe ser entendida como un proceso dinámico, de apertura y mestizaje.

\section{Diseño y metodología}

El problema de investigación que nos planteamos al inicio del estudio era: ¿en qué medida se siente integrado ${ }^{4}$ el alumnado de origen marroquí de educación obligatoria en Andalucía? y ¿cuáles podrían ser las líneas de actuación para favorecer la convivencia escolar? Con el propósito de dar respuesta al problema planteado, el objetivo general de este trabajo es:

- Analizar aquellos factores más relevantes que favorecen la inclusión del alumnado de origen marroquí matriculado en centros de educación primaria públicos de Andalucía.

- La metodología utilizada en la presente investigación es múltiple y diversa, combinando el enfoque cualitativo y cuantitativo. Nos apoyamos en varias técnicas de recogida de información, a saber: el análisis documental, el cuestionario y la observación participante. Para el análisis de los datos obtenidos con la segunda técnica mencionada, hemos utilizado el programa estadístico SPSS. Mientras que en la observación hemos empleado los registros anecdóticos. El trabajo de campo se ha desarrollado durante los años 2009 y 2010.

\footnotetext{
3- $<$ http://portal.unesco.org/es/ev.php-URL_ID=13179\&URL_D0=D0_ TOPIC\&URL_SECTION=201.html>

4- Este término es entendido en el presente trabajo desde un enfoque inclusivo, yendo más allá del modelo de gestión basado en la asimilación y la homogeneización. Sin embargo, para facilitar la lectura, haremos uso de ambos términos (integración-inclusión) como sinónimos.
} 
Entre las técnicas de recogida de información, el cuestionario se ha consagrado como el principal instrumento. El diseño del mismo ha sido un proceso largo y riguroso que ha atravesado una serie de períodos desde su selección hasta su construcción, de forma que pudiéramos garantizar con ello su efectividad. La finalidad principal del mencionado instrumento ha sido conocer la opinión del alumnado objeto de estudio sobre su integración en el colegio con los distintos agentes educativos que participan y en el barrio. La cédula final quedó conformada con 32 ítems.

La observación participante la entendemos como un proceso de interacción entre el observador y el contexto de estudio, sirviéndonos para complementar e interpretar mejor la información obtenida a través del cuestionario. En el caso que nos ocupa, el rol del observador ha sido moderado (SPRADLEY, 1980).

\section{Estimación y tamaño muestral}

Utilizamos el muestreo polietápico estratificado por conglomerado. Para la determinación del tamaño de los estratos nos servimos del criterio de asignación proporcional. De esta forma, tal y como plantean Rodríguez, Garía y Gil, "respetaríamos la importancia cuantitativa de cada estrato, asignando en la muestra un número de individuos proporcional al tamaño del estrato en la población” (1995, p. 221).
Población de estudio para el alumnado de origen marroquí de educación primaria.

a) Población: alumnado de nacionalidad marroquí matriculado en los centros públicos de educación primaria de la Comunidad Autónoma de Andalucía.

b) Población de referencia: la población tomada como referencia es la facilitada por la Consejería de Educación de la Junta de Andalucía para el curso 2008/2009.

La variable elegida para la estratificación es la provincia donde se encuentra matriculado el alumnado, pues vamos a utilizar el muestreo aleatorio estratificado.

Según los datos ofrecidos por la Consejería de Educación de la Junta de Andalucía, en el curso indicado había un total de 6.370 alumnos procedentes de Marruecos matriculados en los centros de enseñanza públicos de Andalucía. Teniendo en cuenta la población total y siguiendo a Sierra Bravo (1985), para un error muestral de aproximadamente un 4\% la muestra representativa de dicho colectivo debería estar compuesta por 588 sujetos. Puesto que empleamos el criterio de asignación constante, cada uno de los estratos contribuyen a la muestra con un número de individuos equitativo al tamaño del estrato en la población. Para ello aplicamos la siguiente fórmula: $\mathrm{n}_{1}=\mathrm{n} \cdot \mathrm{N}_{1} / \mathrm{N}^{5}$.

En la siguiente tabla presentamos la población de referencia y los cálculos realizados para obtener la muestra en cada uno de los estratos:

Tabla 1: Población y muestra por estratos

\begin{tabular}{|c|c|c|c|}
\hline Provincia & $\begin{array}{c}\text { Alumnado } \\
\text { matriculado }\end{array}$ & $\mathrm{n}_{1}=\mathrm{n} \cdot \mathrm{N}_{1} / \mathrm{N}$ & $\begin{array}{c}\text { Muestra por } \\
\text { estratos }\end{array}$ \\
\hline Almería & 2531 & $\mathrm{n}_{\text {Almería }}=\mathrm{n} \cdot \mathrm{N}_{1} / \mathrm{N}=588.2531 / 6370$ & 234 \\
\hline Cádiz & 516 & $\mathrm{n}_{\text {Cádiziz }}=\mathrm{n} \cdot \mathrm{N}_{\mathrm{1}} / \mathrm{N}=588 \cdot 516 / 6370$ & 48 \\
\hline Córdoba & 232 & $\mathrm{n}_{\text {Córdoba }}^{\text {Cála }}=\mathrm{n} \cdot \mathrm{N}_{1} / \mathrm{N}=588 \cdot 232 / 6370$ & 21 \\
\hline Granada & 580 & $n_{\text {Granada }}=n \cdot N_{1} / N=588 \cdot 580 / 6370$ & 53 \\
\hline Huelva & 417 & $\mathrm{n}_{\text {Huelara }}=\mathrm{n} \cdot \mathrm{N}_{1} / \mathrm{N}=588.417 / 6370$ & 39 \\
\hline Jaén & 328 & $\mathrm{n}_{\text {Jaén }}=\mathrm{n} \cdot \mathrm{N}_{1} / \mathrm{N}=588 \cdot 328 / 6370$ & 30 \\
\hline Málaga & 1350 & $n_{\text {Malaga }}=n \cdot N_{1} / N=588 \cdot 1350 / 6370$ & 125 \\
\hline Sevilla & 416 & $\mathrm{n}_{\text {Sevillaa }}=n \cdot N_{1} / \mathrm{N}=588 \cdot 416 / 6370$ & 38 \\
\hline TOTAL & 6370 & & 588 \\
\hline
\end{tabular}

Fuente: elaboración propia

Datos y perfil de la muestra

5- Siendo: $n_{1} n_{2} n_{3} \quad n_{8}$ las muestras para cada uno de los estratos (provincias); $n$, la muestra representativa para la población objeto de estudiada. $N_{1}$, los tamaños respectivos de las subpoblaciones en cada provincia. N, es el tamaño de la población. 
A continuación presentamos el perfil sociodemográfico y educativo del alumnado de origen marroquí que compone la muestra de estudio, para ello analizaremos la edad, el sexo, la zona de procedencia y el tiempo de estancia en España.

Mayoritariamente el alumnado tiene edades comprendidas entre los 9 y 12 años (69\%), mientras que el $14,6 \%$ se encuentra en los intervalos de edad de 5 a 8 años y el 7,8\% tiene más de 12 años. La inmensa mayoría $(81,9 \%)$ está matriculada en $3^{\circ}, 4^{\circ}, 5^{\circ}$ y $6^{\circ}$ de primaria, concretamente el 17,5\% cursa $3^{\circ}$, el $14,1 \% 4^{\circ}$, el $20,9 \% 5^{\circ}$ y el $29,4 \% 6^{\circ}$. De $1^{\circ}$ y $2^{\circ}$ curso tan solo han contestado el 4,1\% y el 4,6\% respectivamente. Tras las entrevistas realizadas con los directores de los centros, jefes de estudio, orientadores y/o mediadores culturales en la fase de elaboración y validación del instrumento principal de recogida de información, vislumbrábamos que el alumnado objeto de estudio se centraba fundamentalmente en los cursos más avanzados. Esta información nos permitió diseñar un cuestionario para estudiantes que supiesen leer, aunque con preguntas muy sencillas y claras.

Respecto al sexo de los encuestados debemos indicar que la muestra es equitativa, el 50,7\% son niños y el 48,8\% niñas (el 0,5\% restante no ha contestado a este ítem).

La zona de procedencia familiar en Marruecos es otra variable a destacar en este apartado, pues nos permite identificar si existen focos de emigración marroquí. Tras analizar la información recogida, observamos que el $42,6 \%$ es originario de tres regiones consideradas como focos de emigración a Andalucía, ya puesto de manifiesto en diversos estudios (LÓPEZ; BERRIANE, 2004; TERRÓN, 2008). Concretamente, el 19,9\% proviene de la región de Tánger-Tetuán, el 12,2\% Rabat-SaléZemmour-Zaer y el 10,5\% de Tadla-Azilal.

El tiempo de estancia en España es otra variable de crucial importancia a la hora de estudiar la integración del alumnado extranjero. Aspectos tales como el conocimiento de la lengua, cultura, religión de la sociedad de llegada, pueden estar íntegramente relacionados con la concepción que tiene el alumnado de su propia identidad. Aunque somos conscientes que la identidad está en continua construcción, máxime a esas edades. En ella influyen multitud de elementos, conformándose la personalidad de cada uno de nosotros a partir de múltiples pertenencias (MAALOUF, 2009).

En relación a esta variable, algo más de la mitad de la población encuestada $(53,2 \%)$ lleva viviendo en España más de cinco años. El 27\% de dos a cinco años y tan solo el 6,1\%, un año o menos. Por tanto, entendemos que la información recogida respecto a la perspectiva de su integración en España dará respuesta a los objetivos marcados en los inicios de esta investigación, pues ya cuentan con un tiempo prudencial de residencia en España.

\section{Análisis y discusión de resultados}

En el desarrollo de este epígrafe exponemos los resultados más relevantes obtenidos tras la aplicación del cuestionario a la muestra seleccionada en las ocho provincias andaluzas, así como la información recogida durante el proceso de observación. A continuación presentamos, en la tabla 2, las dos dimensiones generales que consideramos fundamental explorar con los respectivos indicadores.

Por un lado, las distintas manifestaciones de la diversidad a través de la lengua -materna y del país de llegada-, religión, costumbres tradicionales, sentimiento de pertenencia identidad-, tipo de relación con iguales, etc. Y, por otro lado, el conocimiento de las medidas y recursos existentes para gestionarla en el contexto estudiado, nos permitirá tener una visión global sobre la percepción del alumnado respecto a su participación como ciudadano en la sociedad donde vive. Ciertamente, tal y como ponen de manifiesto diversos autores, las políticas de un Estado hacia la población inmigrante y los derechos que se les conceda, perfilarán el tipo inserción de estos colectivos en una sociedad determinada. 
Tabla 2: Dimensiones e indicadores

\begin{tabular}{|c|c|}
\hline Dimensiones & Indicadores \\
\hline $\begin{array}{l}\text { Opinión del alumnado sobre algunos } \\
\text { elementos que influyen en su integración }\end{array}$ & $\begin{array}{l}\text { Práctica de las fiestas tradicionales de la cultura de origen y del país de llegada } \\
\text { Idioma utilizado para comunicarse en el hogar familiar } \\
\text { Principales obstáculos al llegar a España. } \\
\text { Sentimiento de pertenencia respecto a la identidad nacional-español y/o marroquí- } \\
\text { Preferencia de los padres respecto a los compañeros de juego } \\
\text { Compañeros de juego en el recreo } \\
\text { Problemas de integración en el barrio y en el colegio }\end{array}$ \\
\hline $\begin{array}{l}\text { Percepción del alumnado sobre los } \\
\text { recursos de la escuela ante la diversidad }\end{array}$ & $\begin{array}{l}\text { Recursos de la escuela ante la diversidad cultural } \\
\text { Profesor de lengua castellana en la escuela } \\
\text { Actividades sobre culturas de otros países } \\
\text { Conocimiento del alumnado autóctono sobre la cultura marroquí } \\
\text { Formación religiosa islámica }\end{array}$ \\
\hline
\end{tabular}

Fuente: elaboración propia

Con la primera dimensión de estudio, nuestro objetivo ha sido conocer la opinión del alumnado sobre determinados elementos que les han podido influir en su integración en la sociedad de llegada, acotándolos a sus contextos más próximos - familia, escuela y barrio.

En esta línea, les formulamos los items: ¿Sueles practicar las fiestas tradicionales de tu país (Fiesta del Cordero, Ramadán? y ¿Sueles practicar las fiestas tradicionales de
España (Navidad, Semana Santa)? Como puede observarse en la siguiente tabla, la inmensa mayoría del alumnado objeto de estudio practica las fiestas tradiciones de su cultura de origen mientras que en el caso de las celebraciones tradicionales del país de llegada - España no existe tanta unanimidad en las respuestas ofrecidas. Algo más de la mitad de la muestra suele celebrar la Navidad, Semana Santa, etc., mientras que un representativo $36,6 \%$ no lo hace.

Tabla 3: Práctica de las fiestas tradicionales de la cultura de origen y del país de llegada

\begin{tabular}{|c|c|c|c|c|c|}
\hline \multicolumn{3}{|c|}{ Fiestas tradicionales de Marruecos } & \multicolumn{3}{|c|}{ Fiestas tradicionales de España } \\
\hline & Frecuencia & Porcentaje (\%) & & Frecuencia & Porcentaje (\%) \\
\hline Sí & 556 & 94,6 & Sí & 365 & 62,1 \\
\hline No & 25 & 4,3 & No & 215 & 36,6 \\
\hline $\mathrm{Ns} / \mathrm{Nc}$ & 7 & 1,2 & $\mathrm{Ns} / \mathrm{Nc}$ & 8 & 1,4 \\
\hline Total & 588 & 100 & Total & 588 & 100 \\
\hline
\end{tabular}

Fuente: elaboración propia a partir de los datos de la investigación

La sociedad española $y$, por consiguiente, sus escuelas se caracterizan por ser pluriculturales, pero las personas de las culturas minoritarias, especialmente de aquellas que no comparten las mismas costumbres, religión que las del país de llegada ¿se sienten igualmente partícipes? Como ciudadanos de un estado aconfesional (CONSTITUCIÓN ESPAÑOLA, 1978, Art. 16.3) la respuesta a la pregunta planteada es obvia en lo referente a la religión; sin embargo, en la práctica diaria no lo es tanto. Debido a nuestra historia y tradición, las principales fiestas culturales a nivel nacional tienen su origen en la religión católica. En este sentido, coincidimos con Eva María González (2008) al afirmar que desde un enfoque intercultural las escuelas deberían abrirse a las distintas formas de ver, sentir y comprender la realidad, convirtiéndose los centros en espacios dinámicos. 
Con este planteamiento defendemos que las costumbres culturales se pueden y deben celebrar en las instituciones educativas, pero sería interesante repensar la forma de abordarlas con el alumnado, con el propósito de que todas las culturas que forman parte de una escuela se sientan representadas para conseguir evitar la discriminación de determinados colectivos. Conocer al otro, su cultura, tradiciones debe ser uno de los primeros pasos para el encuentro intercultural, con el propósito de alcanzar una verdadera convivencia en la sociedad y, especialmente, en la escuela.

Beatriz Macías, Jose Antonio Sánchez y María Cabillas (2011) han observado en las investigaciones que han realizado sobre escuelas multiculturales, que aquellas experiencias educativas que introducen la diversidad escolar en el centro a través de las propias experiencias concretas del alumnado favorecen la integración.

En este tipo de prácticas se produce un equilibrio y un continuo subir y bajar desde/entre lo concreto (la circunstancia personal del estudiante) y lo abstracto (la definición de un grupo cultural) que facilita el conocimiento y empoderamiento de los grupos culturales evitando $\mathrm{su}$ encapsulamiento en estereotipos rehificados. Las experiencias de las personas focalizadas son dinámicas $\mathrm{y}$ diversas, además de subjetivas, por lo que la interpretación e imagen de las culturas proyectada no podrá nunca ser única ni homogeneizante. (p. 646).

Otra de las preocupaciones principales en las que se han centrado muchos trabajos de investigación sobre alumnado extranjero, ha sido la lengua (NAVARRO, 2005; ETXEBERRIA, 2005; GRAÑERAS et al, 2007; ARROYO, 2012) como instrumento clave para favorecer la integración, centrándose tanto en la necesidad de aprender la lengua del país de llegada como en la relevancia de reforzar el mantenimiento de la lengua materna. Félix Etxeberría y Kristina Elosegui (2010), ponen de manifiesto que uno de los factores que dificultan la normalización del alumnado extranjero en los centros escolares de España es el desconocimiento de la lengua vehicular de la escuela. "El alumnado inmigrante, al no entender el idioma, tiende a aislarse y, en muchos casos, a no manifestar su situación de desconcierto, para no molestar o evitar sentirse ridículos por no hablar bien el idioma” (ETXEBERRÍA; ELOSEGUI, 2010, p. 241). A lo que debemos añadir que el alumnado para dominar la lengua de la escuela tarda una serie de años, se trata de un proceso complejo y así lo han demostrado diversos investigadores en sus estudios (CUMMINS, 1981; RAMÍREZ, 1992; THOMAS; COLLIER, 1997).

En la investigación que hemos desarrollado se confirma, en parte, lo expuesto anteriormente. Los datos obtenidos ${ }^{6}$ ponen de manifiesto que el mayor obstáculo que el alumnado ha encontrado al llegar a España en su proceso de integración es la lengua (42,2\%), al que le siguen los amigos (26,2\%). Mientras que las costumbres y la religión son reconocidos por un porcentaje mucho más reducido de alumnado, 7,3\% y 4,8\% respectivamente. En cambio, el 5,3\% del alumnado no ha tenido ningún obstáculo en su integración.

Tal y como se ha demostrado en investigaciones realizadas en el ámbito anglosajón así como en el caso de España, el tiempo de estancia del alumnado extranjero es una variable muy importante a tener en cuenta para la adquisición de las habilidades lingüísticas del alumnado inmigrante, en la comprensión lectora y máxime en el dominio de la escritura, a la que debemos añadir la complejidad del proceso (HUGUET; NAVARRO; JANÉS, 2007).

Estos datos deben hacernos reflexionar sobre las políticas educativas que se vienen aplicando en los centros escolares para favorecer la integración y concretamente, en el caso

6- Tras formularle al alumnado el ítem ¿Cuál fue el mayor obstáculo que encontraste a tu llegada a España? 
que nos ocupa, la adquisición de la lengua de acogida, pues hoy en día sigue siendo éste uno de los principales retos que presenta nuestro sistema educativo en relación a la atención a la diversidad cultural. Especialmente si los progenitores desconocen sustancialmente la lengua vehicular de la escuela. Hecho que se da de forma más habitual de lo deseado en el colectivo estudiado que proviene de un entorno rural de Marruecos. Consecuentemente, se ralentiza el proceso de integración debido a las limitaciones que pudieran darse en la comunicación entre la familia y la escuela, principales instituciones socializadoras del menor.

Según los datos del informe del Programa Internacional para la Evaluación de Estudiantes (PISA) 2009 sigue existiendo una brecha en el rendimiento académico entre el alumnado nativo e inmigrante, aunque algunos países han sido capaces de reducir esa brecha de forma significativa en estos últimos años, a saber: Bélgica y Suiza. Estas desventajas están muy influenciadas por el nivel socioeconómico de la familia de los menores, por ello es importante implementar por parte de los gobiernos y las instituciones competentes aquellos mecanismos que ayuden a minimizar las desigualdades existentes (PISA IN FOCUS, 2011). Jose Ignacio Vila (2008) en su estudio desarrollado en Cataluña con 567 alumnos y 434 alumnas de origen autóctono y extranjero demuestra que existen determinados factores que reducen o incluso pueden hacer desaparecer las diferencias existentes entre el alumnado de origen autóctono y el extranjero, entre ellos se encuentra el nivel educativo y socioprofesional de las familias y la escolarización desde el inicio del parvulario del alumnado extranjero, evitándose las concentraciones no naturales de este alumnado en determinadas aulas.

El idioma que se habla en el hogar también es considerado como otra variable que influye en el rendimiento del alumnado, si es o no el mismo en el que se realizan las pruebas académicas, así como en la adquisición de la habilidad lingüística de la escuela. En este sentido, en nuestra investigación les preguntamos por el idioma que suelen utilizar en casa para comunicarse. Aproximadamente la mitad de los menores $(43,4 \%)$ expresan que suelen hablar con su familia en árabe, mientras que otro porcentaje similar (43\%) indica que hablan tanto en español como en árabe indistintamente. Además, debemos considerar que el alumnado marroquí puede ser de origen árabe o bereber, heterogeneidad que también se refleja en su lengua materna.

Tabla 4: Idioma con el que se comunican en sus hogares

\begin{tabular}{c|cccc}
\hline Lengua & Frecuencia & Porcentaje (\%) \\
\hline Español & $\vdots 1$ & 41 & 7,0 \\
\hline Árabe & $\vdots$ & 255 & $\vdots$ & 43,4 \\
\hline Francés & $\vdots$ & 2 & $\vdots$ & 0,3 \\
\hline Español y Árabe & $\vdots$ & 253 & $\vdots$ & 43,0 \\
\hline Español y Francés & $\vdots$ & 6 & $\vdots$ & 1,0 \\
\hline Árabe y Francés & $\vdots$ & 4 & $\vdots$ & 0,7 \\
\hline Los 3 idiomas & $\vdots$ & 24 & $\vdots$ & 4,1 \\
\hline Ns/Nc & $\vdots$ & 3 & $\vdots$ & 0,5 \\
\hline Total & $\vdots$ & 588 & $\vdots$ & 100 \\
\hline
\end{tabular}

* No sabe/No contesta

Fuente: elaboración propia a partir de los datos obtenidos en la investigación

Por tanto, la variable lengua familiar, además de la del tiempo de estancia comentada anteriormente, mediatiza el desarrollo de la lengua de la escuela por parte del alumnado inmigrante. La investigación desarrollada por Ángel Huguet, José Luis Navarro y Judit Janés (2007) en los centros públicos de la provincia de Huesca (España) con alumnado de origen inmigrante ha constatado:

(...) un conocimiento lingüístico más bajo en los inmigrantes, considerados en su conjunto, respecto a sus pares autóctonos de igual edad y nivel escolar. Y si estas diferencias son notables en el caso de los niños y niñas hispanoamericanos, todavía lo son más cuando nos referimos a los no-hispanoamericanos. Además, como podía esperarse, al contrastar 
ambos grupos, se aprecian puntuaciones relativamente más altas en los primeros que en los segundo. (p. 375).

Uno de los temas más importantes que completan el bloque de integración es el relacionado con la identidad del alumnado. María Ángeles Marín (2002) plantea que el término identidad tiene un doble significado. En un primer nivel entendido como singularizar.

Desde esta perspectiva, la identidad cultural significa la ubicación propia y la de otros grupos en referencia a una cultura, la clasificación de una persona como perteneciente a un grupo que se supone tiene una cultura específica. (MARÍN, 2002, p. 34).

Mientras que en el segundo nivel planteado, alude a la representación que posee el sujeto.

Desde esta perspectiva, la identidad de los pueblos remite a su cultura, como un sistema de creencias, actitudes y comportamientos que les son comunicados a cada miembro del grupo por su pertenencia a él; es un modo de sentir, comprender y actuar en el mundo y en formas de vida compartidas que se expresan en instituciones, comportamientos regulados, artefactos, objetos artísticos, saberes transmitidos, etc. (MARÍN, 2002, p. 34) .

Partiendo de estas premisas, en nuestra investigación les presentamos el siguiente dilema ¿cómo te sientes más, español y/o marroquí? Según las respuestas ofrecidas, la mayoría de alumnos $(71,6 \%)$ se define como español y marroquí, tan solo el 13,9\% lo hace solo como español, que, debemos aclarar, coincide en gran parte con el alumnado nacido en España. El 11,6\% se define como marroquí, el 0,5\% como otro.

Sin embargo, los resultados obtenidos en nuestra investigación confirman la definición ofrecida por Amin Maalouf (2009), en la que defiende que la identidad no es una foto fija y está en continua construcción, definida por infınidad de elementos que se irán sumando en el desarrollo de nuestras vidas y que entre todos nos harán individual y único. En el caso que nos ocupa, la experiencia de vivir en otro país y entrar en relación con otros grupos, con todo lo que ello conlleva - compartir experiencias, conocer otra cultura, costumbres, idioma, valores, etc.-, ha enriquecido la identidad de gran parte del alumnado estudiado hasta el punto de que, desde su propia perspectiva, se definen con identidades plurales ubicándose a sí mismo en las dos culturas, la marroquí y la española.

Esta información es desde nuestro punto de vista muy importante y nos lleva a coincidir con Amelia Barquín (2009) al afirmar que la identidad múltiple favorece la cohesión social pues hace que los individuos se sientan ciudadanos de la sociedad en la que viven. Si bien, tal y como expone Barquín, las identidades múltiples se deben ir construyendo y en el ámbito escolar se precisa de una intervención pedagógica determinada por parte de los educadores.

Para seguir profundizando en cuestiones relacionadas con la integración de los menores, les preguntamos si sus padres preferían que jugasen con chicos españoles, chicos de origen marroquí o les era indiferente. Es significativo destacar que los padres de la mayoría del alumnado encuestado, no tiene preferencia por ninguna nacionalidad en lo que se refiere a las amistades de sus hijos, al menos así lo manifiesta el $83,2 \%$ de la muestra estudiada. El 10,4\% se decanta por chicos españoles y un 5,3\% expresa que con niños originarios de Marruecos. De forma generalizada advertimos que los padres del alumnado estudiado comparten la idea de preferir que sus hijos e hijas se relacionen en el ámbito académico con todos los menores, pues consideran que ello facilitará su integración en el ámbito escolar, proyectándose normalmente, a la sociedad.

Seguidamente les planteamos el siguiente ítem: En el recreo ¿con quien sueles jugar? De 
los datos obtenidos se desprende que el grupo objeto de estudio suele jugar en el recreo tanto con niños españoles como marroquíes (27\%), así como también se observa un porcentaje similar $(23,3 \%)$ de niños que juegan con sus compañeros sin determinar la procedencia. Información que pudimos contrastar y confirmar con nuestras observaciones.

De esta situación puede deducirse que existe poco rechazo hacia otras culturas entre el alumnado de la escuela primaria de Andalucía, lo cual se relaciona con el alto porcentaje de niños marroquíes que no percibe problemas de integración en el colegio (71,6\%), como se verá posteriormente. Este escenario puede ser también producto del fomento, por parte de las escuelas, de compartir conocimiento acerca de otras culturas, como veremos más adelante. Sin embargo, existe un porcentaje significativo $(13,1 \%)$ de niños que juegan sólo con compañeros marroquíes, pudiendo ser esto debido a dos motivos principalmente: a) Que estos menores tienen un menor grado de integración en los colegios que estudian, lo que puede deberse, por ejemplo, a que buscan espacios donde hablar el idioma materno o expresar aspectos culturales compartidos y/o b) Que en determinados centros exista una concentración más importante de alumnado de nacionalidad marroquí, como pudimos observar en determinados colegios de Almería.

Tabla 5: Compañeros de juego en el recreo

\begin{tabular}{|c|c|c|}
\hline Nacionalidad & Frecuencia & Porcentaje (\%) \\
\hline Españoles & 137 & 23,3 \\
\hline Marroquíes & 77 & 13,1 \\
\hline De otros países & 67 & 11,4 \\
\hline Españoles y marroquíes & 159 & 27,0 \\
\hline Españoles y de otros países & 1 & 0,2 \\
\hline Marroquíes y de otros países & 2 & 0,3 \\
\hline Con todos & 137 & 23,3 \\
\hline $\mathrm{Ns} / \mathrm{Nc}^{*}$ & 8 & 1,4 \\
\hline Total & 588 & 100 \\
\hline
\end{tabular}

Para finalizar la primera dimensión de estudio sobre la opinión del alumnado en relación a determinados elementos que influyen en su integración, les preguntamos si han tenido problemas de integración en su barrio y/o en el colegio por su condición de extranjero. En ambos casos, la mayoría de niños encuestados manifiesta no haber tenido problemas de integración por ser extranjero (72,6\% en el barrio y 71,6\% en el colegio). Sin embargo, existe un número importante de alumnos que han tenido problemas por esa causa en su núcleo residencial $(18,9 \%)$ y en el centro escolar (19,9\%).

Antes de adentrarnos a analizar la información derivada de los ítems planteados en la segunda dimensión de estudio, -percepción del alumnado sobre los recursos de la escuela ante la diversidad-, consideramos oportuno reflexionar sobre la importancia que tienen las políticas puestas en marcha y los recursos disponibles para gestionar adecuadamente la diversidad cultural. Entendemos que una gestión apropiada pasa por dar respuesta educativa de calidad y con equidad a todo el alumnado matriculado en los centros educativos. Tal y como afirma Francesc Carbonell (2002), para desarrollar una educación intercultural además de una serie de factores coyunturales existen otros factores estructurales, no menos importantes, que debemos tener en consideración.

Los problemas de convivencia multicultural no son sólo un problema educativo, son un problema político de hondo calado. Hace falta una voluntad, una actitud y unas actuaciones políticas decididas a dedicar los recursos materiales y humanos que hagan falta para luchar con eficacia contra las causas y las consecuencias de la exclusión social. (p. 64).

En España, las competencias en materia educativa están transferidas a las Comunidades Autónomas, siendo el estado el que salvaguarda la homogeneidad y unidad del sistema educativo. 
Concretamente, la Comunidad Autónoma de Andalucía tiene asumida plenamente dichas competencias a través de la Consejería de Educación de la Junta de Andalucía. A la que le corresponde, según el Ministerio de Educación, Cultura y Deporte, el desarrollo de las políticas estatales básicas que marcan las directrices generales sobre la política educativa, competencias ejecutivo-administrativas, asumiendo las funciones, servicios y recursos en esta materia dentro de su territorio.

En Andalucía, la normativa con referencia directa a la atención educativa a la diversidad, centrándonos especialmente en aquella que alude a la cultura, con independencia de los referentes legales de alto rango $^{7}$, es la siguiente:

Tabla 6: Marco normativo en Andalucía para la atención a la diversidad cultural

\begin{tabular}{lll}
\hline Ley 9/1999, de 18 de noviembre de & $\begin{array}{l}\text { Con esta ley la Junta de Andalucía pretende fortalecer su responsabilidad para la integración social } \\
\text { 1999, de Solidaridad en Educación }\end{array}$ & $\begin{array}{l}\text { y compensar las desigualdades existentes en la escolarización del alumnado para conseguir el tan } \\
\text { deseado Estado de Bienestar, a través de programas y actuaciones de compensación educativa y } \\
\text { social. }\end{array}$ \\
\hline Decreto 167/2003 de 17 de junio. & $\begin{array}{l}\text { Se articulan medidas y actuaciones compensatorias cuyos principales destinatarios son el alumnado } \\
\text { que se encuentre en desventaja sociocultural, siendo uno de los colectivos destinatarios el alumnado } \\
\text { perteneciente a familias inmigrantes. }\end{array}$ \\
\hline Orden de 15 de enero de 2007. & $\begin{array}{l}\text { Entre sus principales objetivos se encuentra la regulación de las actuaciones educativas destinadas } \\
\text { a la educación intercultural por parte de los centros escolares de Educación Primaria y Educación } \\
\text { Secundaria Obligatoria. Especialmente, las Aulas Temporales de Adaptación Lingüística. }\end{array}$ \\
\hline Orden de 25 de junio de 2008 & $\begin{array}{l}\text { Regula y desarrolla medidas y programas de atención a la diversidad del alumnado en educación } \\
\text { básica de Andalucía. }\end{array}$ \\
\hline
\end{tabular}

Fuente: elaboración propia

En este sentido debemos ser conscientes de que los centros docentes en nuestro contexto de estudio tienen autonomía para organizar la atención a la diversidad de su alumnado, garantizando que todos los estudiantes obtengan el máximo desarrollo posible de las capacidades personales. La institución educativa podrá adoptar las medidas educativas que considere necesarias entre las ofertadas por la Comunidad Autónoma dependiendo de las particularidades educativas del alumnado. Dichas medidas deberán formar parte del proyecto de centro, lo que hace que no existan dos centros educativos iguales.

Una vez realizada esta aproximación al contexto normativo, pasamos a analizar los resultados obtenidos sobre la percepción que el alumnado tiene de los recursos de su escuela ante la diversidad.

Uno de los ítems planteados que integran este bloque es ¿tuviste algún profesor/a en la escuela para aprender español? En este caso la mayoría de los menores marroquíes encuestados $(67,5 \%)$ afirman haber tenido un docente en el aprendizaje de español, frente a un 26,4\% que señalan que no han contado con ningún profesional dedicado a este fin. Como vimos anteriormente, el aprendizaje de la lengua de la escuela es muy importante para el rendimiento escolar del menor y, como no podría ser de otra forma, para favorecer la integración. Como hemos explicado anteriormente, estimamos que los centros deben poner a disposición los recursos necesarios, tanto humanos como materiales, para dar una adecuada respuesta a la diversidad existente. El profesorado destinado a enseñar la lengua vehicular de la escuela es uno de ellos. Si bien, en este caso no es suficiente con que un centro disponga de un profesor para tal fin, sino que además se debe velar porque el docente sea competente interculturalmente. La adquisición del español como lengua de instrucción en el alumnado extranjero es un proceso lento y complejo, como ya se ha comentado, y a nivel didáctico, para el caso del alumnado marroquí, deben considerarse una serie de variantes sociolingüísticas y socieducativas que

7- Ley Orgánica 2/2006, de 3 de mayo, de Educación y la Ley 17/2007, de 10 de diciembre, de Educación de Andalucía. 
condicionan sustancialmente dicho proceso de enseñanza-aprendizaje (EL-MADKOURI, 2003).

Con los siguientes ítems pretendemos indagar en el conocimiento y práctica de la cultura tanto la de origen -Marruecos- como la de la sociedad de llegada -España.

Para ello comenzamos preguntándoles si en su colegio practican actividades que traten de culturas de otros países. Según los datos obtenidos, algo más de la mitad de los niños marroquíes de la escuela primaria $(63,1 \%)$ afirma que en su centro escolar se llevan a cabo actividades relacionadas con culturas de otros países. Sin embargo, un $31,5 \%$ señalan que no se realizan actividades de este tipo. En este sentido, debemos completar la información con las observaciones realizadas en el terreno, pues es importante destacar que estas iniciativas generalmente no están presentes todos los días del curso en el centro educativo, sino que son más bien puntuales. No obstante, si debemos destacar que existen muchas escuelas donde el intercambio de conocimientos sobre las distintas culturas está en la vida diaria del centro, con carteles, fotos, mensajes en distintas lenguas, especialmente en aquellas donde el alumnado es más diverso en relación a la cultura de origen.

Otra de las cuestiones que les planteamos es si sus compañeros de distinta nacionalidad conocen aspectos importantes de su cultura marroquí. La mayoría de respuestas son afirmativas (60,7\%), mientras que un 32,3\%, cree lo contrario. Las iniciativas por parte de las escuelas de compartir conocimientos acerca de otras culturas pueden relacionarse con el alto porcentaje de niños marroquíes que dicen que sus compañeros conocen aspectos de su cultura. Si bien, y debido a la importancia que tiene en nuestros días la educación intercultural, nos resulta destacable el porcentaje tan elevado de alumnos que contestan a la pregunta de forma negativa. Lo que nos lleva a pensar que aún queda mucho por hacer en este terreno.

En esta línea, nos gustaría exponer que la educación intercultural no es tener en la programación de actividades académicas la semana intercultural, el día de la gastronomía de un país o de distintos países, no es educación compensatoria ni pretende evitar los conflictos, sino que se trata de un proceso transformador, de una educación para todos, valorando la riqueza que otorga la diversidad, promoviendo la relación entre las personas y aprendiendo de los conflictos. Según Amelia Barquín:

Lo que sí debe hacer la escuela es tener en cuenta y estimar las características culturales propias del alumno y su pertenencia al grupo de origen de su familia, lo cual significa en la práctica proporcionar las circunstancias para que afloren y darles lugar y prestigio en el aula. Que las lenguas de los niños estén presentes en la escuela de diversos modos (carteles, poemas, canciones, etc.), que la escuela aliente a la familia para que no abandone su lengua autóctona con el niño, sino todo lo contrario, que la vida en el hogar y en el grupo de origen familiar pueda ser compartida en clase de modo positivo, que los familiares puedan participar de la vida escolar en distintas formas (colaborando dentro del aula, tomando parte en la vida organizativa del centro, etc.), son algunos ejemplos de ello. (2009, p. 94).

Un aspecto que consideramos de gran interés es la formación religiosa, por ello le preguntamos si reciben actualmente formación religiosa islámica. Es importante destacar que el $79,8 \%$ sí la recibe, y tan solo un 17\% contesta de forma negativa a la pregunta.

Tabla 8: ¿Recibes formación religiosa islámica?

\begin{tabular}{ccc:c}
\hline Formación religiosa & Frecuencia & Porcentaje (\%) \\
\hline Sí, en la escuela y otros espacios & $\vdots$ & 133 & 22,7 \\
\hline Sí, en familia y otros espacios & $\vdots$ & 287 & 48,8 \\
\hline Sí, en asociaciones & $\vdots$ & 37 & 6,3 \\
\hline Sí, con amigos & $\vdots$ & 12 & 2,0 \\
\hline No recibo ninguna formación & $\vdots$ & 100 & 17,0 \\
\hline Ns/Nc* & $\vdots$ & 19 & 3,2 \\
\hline Total & $\vdots$ & 588 & 100 \\
\hline *No sabe/No contesta & \multicolumn{3}{c}{} \\
Fuente: elaboración propia a partir de los datos obtenidos en la investigación
\end{tabular}


Es significativo destacar que de aquellos alumnos que afirman recibir formación religiosa islámica el 48,8\% lo hace en su familia, además de con los amigos. Tan solo el 22,7\% afirma formarse en la escuela entre otros espacio. Estos últimos datos nos parecen relevantes pues la Constitución española (1978) garantiza el derecho de los padres a que sus hijos reciban formación religiosa $\mathrm{y}$ moral, acorde a sus convicciones, en la escuela (Art.27.3). Sin embargo la práctica es una cuestión más compleja. Según exponen Sol Tarrés y F. Javier Rosón (2009), la normativa en materia educativa precisa que les corresponde a los padres solicitar a los centros educativos las clases de religión minoritaria a través de los impresos de preinscripción, y posteriormente estas solicitudes se elevan al Ministerio de Educación. No obstante, debe existir un mínimo de 10 alumnos para que pueda haber un profesor de religión minoritaria en un centro. Sin embargo, existe desconocimiento por parte de los padres respecto a la posibilidad que tienen sus hijos de recibir formación en una determinada religión, a lo que se suma la complicación de la tramitación de dicha solicitud o el cuestionamiento sobre la formación que recibirán por parte del profesor asignado, entre otras limitaciones.

\section{Consideraciones finales}

A continuación pasamos a exponer aquellas conclusiones más significativas derivadas del estudio presentado, para ello tendremos en cuenta las dos grandes dimensiones planteadas en los inicios de nuestro trabajo.

Respecto a la primera dimensión de estudio:

- La inmensa mayoría del alumnado participante en el estudio practica las fiestas tradicionales de su cultura materna (94,6\%). Además de practicar algo más de la mitad de la muestra $(62,1 \%)$ las fiestas tradicionales españolas. Esta información nos hace plantearnos una serie de cuestiones para invitar a la reflexión ¿las escuelas se abren a las distintas formas de sentir y comprender la realidad? ¿dónde y cómo se llevan a cabo este tipo de fiestas? ¿se parte de la experiencia del propio alumnado?

- La adquisición de la lengua de la escuela, a pesar de ser instrumento clave para favorecer la integración, es hoy día para el alumnado marroquí uno de los mayores obstáculos en el proceso de integración $(42,2 \%)$; al que le siguen el hacer amigos (26,3\%), estando este último intimamente relacionado con el primero.

- El idioma que se habla en el hogar es considerado como una variable influyente en el rendimiento académico del alumnado, además del tiempo de estancia en el país de llegada. En nuestro estudio, prácticamente la mitad del alumnado manifiesta hablar en casa en su lengua materna (árabe) y otro porcentaje muy similar hablar tanto en árabe como en español. Por lo que entendemos que éstos últimos pudieran tener más facilidad para adquirir las competencias lingüísticas demandadas en la escuela. En este sentido, debemos resaltar que en algunos casos los padres y madres desconocen la lengua del país de llegada, teniendo más dificultades que los menores para aprenderla.

- La mayor parte del alumnado estudiado afirma tener una identidad múltiple, definiéndose a sí mismo tanto marroquí como español (71,6\%). Desde nuestro punto de vista, esta información es muy relevante pues esta circunstancia puede favorecer en gran medida la cohesión social en la región andaluza. No obstante, y coincidiendo con Amelia Barquín (2009), dicha realidad demanda por parte de los educadores intervenciones pedagógicas planificadas para seguir favoreciendo y madurando las identidades múltiples. Tal y como afirma Vicente Llorent, en la escuela los menores “(...) interiorizan muchos valores y normas de convivencia no sólo de una forma explícita, sino también a través del denominado curriculum oculto, que tendrá indudables repercusiones en sus vidas" (2011, p. 93).

- Algo más de la mitad de la población estudiada $(50,3 \%)$ expone jugar en el recreo 
con sus compañeros sin tener en cuenta la nacionalidad de origen de los mismos. Además la mayoría del alumnado no ha tenido problemas de integración ni en el barrio ni en el colegio (72,6\% y 71,6\% respectivamente).

Respecto a la segunda dimensión planteada; uno de los aspectos importantes a tener en cuenta para analizar si en las escuelas andaluzas está presente la interculturalidad, es conocer si los centros se caracterizan por ser espacios abiertos, donde los distintos agentes sociales y las distintas culturas están presentes diariamente. Esto propiciaría la interacción y el intercambio de la comunidad educativa.

- La religión islámica rige la vida de los que la profesan, por lo que es comprensible la importancia que las familias le otorgan a la formación de la misma para con sus infantes. En esta línea, la mayoría del alumnado manifiesta recibir formación islámica (79,8\%). De los cuales la mayor parte lo hace en el ámbito familiar
$(48,8 \%)$ y $\tan$ solo el $22,7 \%$ en la escuela u otros espacios. En, en base a la Constitución y a los acuerdos firmados con distintas religiones, entre ellas está el Islam, los recursos asignados en las escuelas para la formación de la religión islámica deben seguir mejorando.

- Algo más de la mitad del alumnado afirma que en sus centros se desarrollan actividades relacionadas con otras culturas, aunque no en todos los casos se hace desde la premisa de la educación intercultural, sino más bien desde la educación multicultural con acciones puntuales. No obstante, debemos reconocer que existen centros en la Andalucía verdaderamente interculturales, con proyectos integradores y con personal muy comprometido en educar bajo este modelo pedagógico; coincidiendo en casi todos los casos observados con aquellos en los que la diversidad cultural del alumnado es una característica muy presente.

\section{Referencias}

ARROYO, María José. Las aulas de inmersión lingüística para alumnado inmigrante en el marco de la escuela inclusiva: algunas propuestas de mejora. Tendencias Pedagógicas, Madrid, Universidad Autónoma de Madrid, n. 19, p. 25-42, 2012.

BARQUÍN, Amelia ¿De dónde son los hijos de los inmigrantes? La construcción de la identidad y la escuela. Educar, Barcelona, Universitat Autònoma de Barcelona, n. 44, p. 81-96, 2009

BORRERO, Raquel. Educación para todos y todas. Análisis del modelo educativo-social para una educación intercultural. Educatio Siglo XXI, Murcia, Universidad de Murcia v. 30, n. 2, p. 333-364, 2012.

CARBONELL, Francesc. Educación intercultural: principales retos y requisitos indispensables. Kikiriki, Sevilla, Cooperación Educativa, n. 65, p. 63-68, 2002.

CUMMINS, Jim. Age on arrival and immigrant second language learning in Canada: a reassessment. Applied Linguistics, Oxford, Oxford Journals, v. 2, n. 2, p. 132-149, 1981.

EL-MADKOURI, Mohamed. El mapa lingüístico-educativo marroquí y su influencia en la adquisición del español como lengua de instrucción. Revista Electrónica de Estudios Filológicos, n. 5, 2003. Disponible en: <http://www.um.es/tonosdigital/znum5/ estudios/F-mapalin.htm>. Acceso en: 22 nov. 2013.

ESCARBAJAL, Andrés. Hacia la educación intercultural. Pedagogía Social: Revista Interuniversitaria, A Coruña, Sociedad Iberoamericana de Pedagogía Social, n. 18, p. 131-149, 2011.

ESPAÑA. Constitución española (1978). DerechosHumanos.net. Disponible en: <http://www.derechoshumanos.net/constitucion/ index.htm?gclid=CJ_lzceEjrgCFfLHtAodaA0A2g >. Acceso en: 29 jun. 2013. 
ESPAÑA. Ley 9/1999, de 18 de noviembre, de solidaridad en educación. B0JA, n. 140, p. 15428, 21 jul. 2009.

ESPAÑA. Decreto 167/2003, de 17 de junio, por el que se establece la ordenación de la atención educativa al alumnado con necesidades educativas especiales asociadas a condiciones sociales desfavorecidas. B0JA, n. 118, 23 jun. 2003.

ESPAÑA. Ley orgánica 2/2006, de 3 de mayo, de educación. B0E, n. 106, 4 mayo 2006. (B0E-A-2006-7899).

ESPAÑA. Ley 17/2007, de 10 de diciembre, de educación de Andalucía. B0JA, n. 252, 26 dic. 2007.

ESPAÑA. Orden de 15 de enero de 2007, por la que se regulan las medidas y actuaciones a desarrollar para la atención del alumnado inmigrante y, especialmente, las aulas temporales de adaptación lingüística. B0JA, n. 33, Sevilla, 14 feb. 2007.

ESPAÑA. Orden de 15 de enero de 2007, por la que se regulan las medidas y actuaciones a desarrollar para la atención del alumnado inmigrante y, especialmente, las aulas temporales de adaptación lingüística. B0JA, n. 33, 14 feb. 2007.

ESPAÑA. Orden de 25 de julio de 2008, por la que se regula la atención a la diversidad del alumnado que cursa la educación básica en los centros docentes públicos de Andalucía. B0JA, n. 167, 22 ago. 2008.

ETXEBERRÍA, Félix; ELOSEGUI, Kristina. Integración del alumnado inmigrante: obstáculos y propuestas. Revista Española de Educación Comparada, Madrid, Sociedad Española de Educación Comparada y Universidad Nacional de Educación a Distancia, n. 16, p. 235-263, 2010.

ETXEBERRIA, Félix. Lenguas inmigrantes en la escuela en España. Cuadernos Interculturales, Valparaíso, Universidad de Valparaíso, Año 3, n. 5, p. 9-30, 2005.

GONZÁLEZ, Eva María. Convivencia intercultural en centros educativos multiculturales... ¿Hacia un reconocimiento de la diversidad cultural? Education Siglo XXI, Murcia, Universidad de Murcia, n. 26, p. 225-240, 2008.

GRAÑERAS, Montserrat, et al. La atención lingüística al alumnado extranjero en el sistema educativo español: normativa, actuaciones y medidas. Revista de Educación, Madrid, Ministerio de Educación, Cultura y Deporte, n. 343, p. 149-176, 2007.

HUGUET, Ángel; NAVARRO, José Luis; JANÉS, Judit. La adquisición del castellano por los escolares inmigrantes. El papel del tiempo de estancia y la lengua familiar. Anuario de Psicología, Barcelona, Universidad de Barcelona, v. 38, n. 3, p. 357-375, 2007.

INADI - INSTITUTO NACIONAL CONTRA LA DISCRIMINACIÓN, LA XENOFOBIA Y EL RACISMO. Derecho a la educación sin discriminación. Buenos Aires: Ministerio de Justicia y Derechos Humanos, 2012.

JIMÉNEZ, Paco; VILÀ, Montserrat. De educación especial a educación en la diversidad. Málaga: Aljibe, 1999.

LÓPEZ, Bernabé; BERRIANE, Mohamed. Atlas de la inmigración marroquí en España. Madrid: Dirección General de Migraciones: UAM, 2004.

LLORENT, Vicente. Conformación y control del sistema escolar marroquí: del protectorado francés a la independencia. Historia de la Educación: Revista Interuniversitaria, Salamanca, Universidad de Salamanca, n. 30, p. 91-109, 2011.

MAALOUF, A. Identidades asesinas. Madrid: Alianza, 2009. 5. reimpresión.

MACÍAS, Beatriz; SÁNCHEZ, José A.; CABILLAS, María. Etnografía de la interculturalidad: analizando buenas prácticas en escuelas multiculturales andaluzas. In: GARCÍA, Francisco Javier; KRESSOVA, Nina (Coords.). CONGRESO INTERNACIONAL SOBRE MIGRACIONES EN ANDALUCÍA, 1., Granada, 2011. Actas de I I... Granada: Instituto de Migraciones, 2011. p. 639-648.

MARÍN, María Ángeles. Construcción de la identidad en la época de la mundialización y los nacionalismos. In: BARTOLOMÉ, Margarida (Coord.). Identidad y ciudadanía: un reto a la educación intercultural. Madrid: Narcea, 2002. p. 27-50.

NAVARRO, José Luis. Inmigración en España y conocimiento de la lengua castellana: el caso de los escolares inmigrados en Aragón. Lleida: Universitat de Lleida, 2005. 369 p. Tesis (Doctoral) - Universitat de Lleida. Facultad de Ciencias de la Educación. Lleida, 2005. 
OPAM - OBSERVATORIO PERMANENTE ANDALÚZ DE LAS MIGRACIONES. Alumnado extranjero en enseñanza no universitaria en Andalucía y provincias: avance curso 2011-2012. Junta de Andalucía. Disponible en: <http://www.juntadeandalucia.es/ justiciaeinterior/opam/?q=peb_hist\&id_peb=94>. Acceso en: 18 jun. 2013.

PISA IN FOCUS ¿Cómo se están adaptando los sistemas escolares al creciente número de estudiantes inmigrantes? Pisa in Focus, OCUS, OCDE, v. 11, dic. 2011. Disponible en: <http://www.oecd.org/pisa/pisaproducts/49861560.pdf>. Acceso en: 29 jun. 2013.

PULID0, Rafael. Diversidad, diferencia, desigualdad y discriminación: una propuesta para ordenar los discursos anti-racistas en educación. Granada: Consejería de Gobernación, 1997.

RAMÍREZ, J. D. Executive summary. Bilingual Research Journal, Philadelphia, National Association for Bilingual Education, v. 16, p. 1-62, 1992.

RODRIGUEZ, Gregorio; GARCÍA, Eduardo; GIL, Javier. Estadística básica aplicada a las ciencias de la educación. Sevilla: Kronos, 1995.

SIERRA BRAVO, Restituto. Técnicas de investigación social: teoría y ejercicios. Madrid: Paraninfo, 1985.

SPRADLEY, James P. Participant observation. New York: Holt: Rinehart and Winston, 1980.

TARRÉS, Sol; ROSÓN, F. Javier. La enseñanza de las religiones minoritarias en la escuela: el caso de Andalucí. 'llu. Revista de Ciencias de las Religiones, Madrid, Universidad Complutense de Madrid, v. 14, p. 179-197, 2009.

TERRÓN, Maria T. Perfil socioeducativo de la inmigración femenina marroquí. Estudio en la provincia de Huelva. Espaço S.: Revista de Investigação e Interevanção Social do ISCE, Ramada, Instituto Superior de Ciências Educativas, v. 2, p. 199-215, 2008.

THOMAS, Wayne. P.; COLLIER, Virginia. School effectiveness for language minority students. Washington, DC: National Clearinghouse for Bilingual Education, 1997.

UNESCO. Declaración universal a favor de la diversidad cultural, 2001. Disponible en: <http://portal.unesco.org/es/ev.phpURL_ID=13179\&URL_DO=D0_TOPIC\&URL_SECTION=201.html>. Acceso en: 30 jun. 2013.

VILA, J. Ignacio. Lengua familiar y conocimiento de la lengua escolar en Cataluña al finalizar la educación infantil. Revista de Educación, Madrid, Ministerio de Educación, Cultura y Deporte, n. 346, p. 401-424, mayo/ago. 2008.

Recibido en: 16.07.2013

Aprobado en: 11.12.2013

Teresa Terrón-Caro es doctora en Pedagogía por la Universidad de Sevilla (España). Profesora del Departamento de Educación y Psicología Social de la Universidad Pablo de Olavide, de Sevilla.

Verónica Cobano-Delgado es doctora en Pedagogía por la Universidad de Sevilla (España). Profesora del Departamento de Teoría e Historia de la Educación y Pedagogía Social de la Universidad de Sevilla. 